\title{
NGTS and WASP photometric recovery of a single-transit candidate from TESS
}

\author{
Samuel Gill, ${ }^{1,2 \star}$ Daniel Bayliss ${ }^{\circledR},{ }^{1,2}$ Benjamin F. Cooke, ${ }^{1,2}$ Peter J. Wheatley ${ }^{\circledR},{ }^{1,2}$ \\ Louise D. Nielsen ${ }^{\odot}, 3$ Monika Lendl ${ }^{\odot}, 3,4$ James McCormac, ${ }^{1,2}$ Edward M. Bryant, ${ }^{1,2}$ \\ Jack S. Acton, ${ }^{5}$ David R. Anderson ${ }^{\oplus}, 1,2$ Claudia Belardi, ${ }^{5}$ François Bouchy, ${ }^{3}$ \\ Matthew R. Burleigh, ${ }^{5}$ Andrew Collier Cameron ${ }^{\oplus}{ }^{6}$ Sarah L. Casewell, ${ }^{5}$ \\ Alexander Chaushev, ${ }^{7}$ Michael R. Goad, ${ }^{5}$ Maximilian N. Günther ${ }^{8} \dagger$ Coel Hellier, ${ }^{9}$ \\ James A. G. Jackman ${ }^{\oplus},{ }^{1,2}$ James S. Jenkins ${ }^{\oplus},{ }^{10,11}$ Maximiliano Moyano, ${ }^{12}$ \\ Don Pollacco, ${ }^{1,2}$ Liam Raynard, ${ }^{5}$ Alexis M. S. Smith, ${ }^{13}$ Rosanna H. Tilbrook, ${ }^{5}$ \\ Oliver Turner ${ }^{\oplus},{ }^{3}$ Stéphane Udry ${ }^{3}$ and Richard G. West ${ }^{\oplus 1,2}$
}

Affiliations are listed at the end of the paper

Accepted 2019 November 13. Received 2019 November 13; in original form 2019 October 11

\begin{abstract}
The Transiting Exoplanet Survey Satellite (TESS) produces a large number of single-transit event candidates, since the mission monitors most stars for only $\sim 27 \mathrm{~d}$. Such candidates correspond to long-period planets or eclipsing binaries. Using the TESS Sector 1 full-frame images, we identified a $7750 \mathrm{ppm}$ single-transit event with a duration of $7 \mathrm{~h}$ around the moderately evolved F-dwarf star TIC-238855958 ( $\left.T_{\mathrm{mag}}=10.23, T_{\text {eff }}=6280 \pm 85 \mathrm{~K}\right)$. Using archival WASP photometry we constrained the true orbital period to one of three possible values. We detected a subsequent transit-event with NGTS, which revealed the orbital period to be $38.20 \mathrm{~d}$. Radial velocity measurements from the CORALIE Spectrograph show the secondary object has a mass of $M_{2}=0.148 \pm 0.003 \mathrm{M}_{\odot}$, indicating this system is an F-M eclipsing binary. The radius of the M-dwarf companion is $R_{2}=0.171 \pm 0.003 \mathrm{R}_{\odot}$, making this one of the most well characterized stars in this mass regime. We find that its radius is $2.3 \sigma$ lower than expected from stellar evolution models.
\end{abstract}

Key words: binaries: eclipsing.

\section{INTRODUCTION}

The Transiting Exoplanet Survey Satellite (TESS, Ricker et al. 2015) has successfully completed its Year 1 survey of the southern ecliptic hemisphere, yielding over 1000 TESS Objects of Interest (TOIs). Already many of these systems have been confirmed as bona fide transiting exoplanets (e.g. Huang et al. 2018; Günther et al. 2019; Nielsen et al. 2019; Vanderburg et al. 2019). The majority of the TOIs are short period systems, with the mean orbital period of these at $7.89 \mathrm{~d}$. This is due to the geometric probability of a transit being inversely proportional to the planet's semimajor axis, and the fact that TESS only monitors most stars for a single $27 \mathrm{~d}$ Sector. In order to discover longer period systems in the TESS data we need to follow-up and characterize the systems

^E-mail: samuel.gill@warwick.ac.uk

$\dagger$ Juan Carlos Torres Fellow. that only present a single-transit event in the TESS light curves. It is clear that there will be a large number of these singletransit candidates in the TESS data (Cooke et al. 2018; Villanueva, Dragomir \& Gaudi 2019), and these longer period systems are scientifically valuable. For low-mass host stars (late K and early M) it will allow us to probe planets in the habitable zones. It is also important to study longer period eclipsing binaries as it allows us to study the mass-radius relationship for low-mass stars without the complicating effects of high stellar irradiation and strong tidal interactions.

With these longer period TESS systems in mind, we have begun a program within the ambit of the NGTS project (Wheatley et al. 2018) to find and characterize single-transit event candidates. This paper reports the first result from this program in which we determine photometrically the orbital period of the TESS singletransit candidate TIC-238855958. We also use spectroscopy to measure the mass of the secondary companion, revealing the system to be a long period F-M binary. 


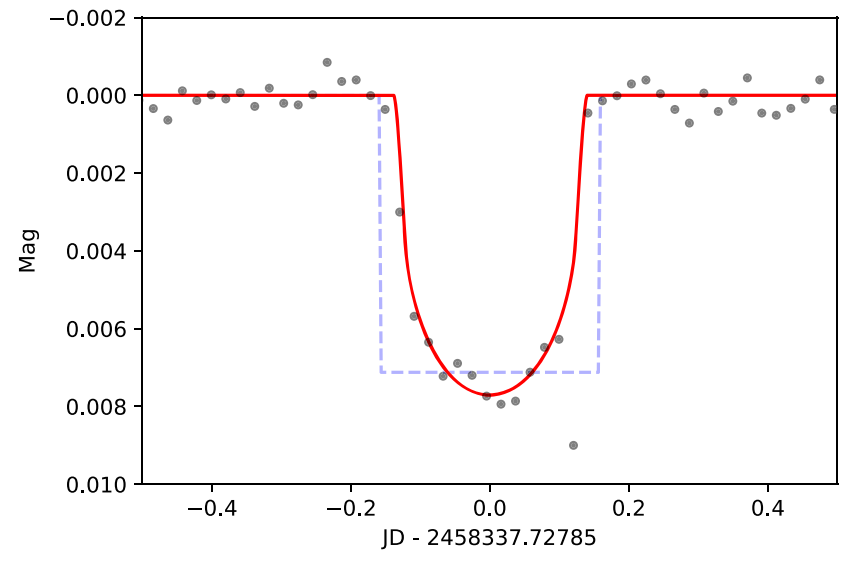

Figure 1. Difference imaging TESS light curve for TIC-238855958 (black) with best-fitting global model (red) and box used to detect the single-transit event (blue dashed).

In Section 2, we outline our single-transit event search of TESS data, which led to the discovery of TIC-238855958. We describe our search of archival WASP photometry in Section 3, and in Section 4 we describe our use of the NGTS facility to recover a subsequent transit of TIC-238855958. We analyse all the data and derive system parameters in Section 6, and finish with our conclusions in Section 7.

\section{SINGLE-TRANSIT EVENT DETECTION}

We conducted a systematic search of TESS light curves for singletransit events. We began by downloading the difference-imaging full-frame light curves produced using the pipeline from Oelkers $\&$ Stassun (2018). These light curves are publicly available on Filtergraph. ${ }^{1}$ We downloaded all light curves with a contamination ratio $<5$. Before beginning our search, we flattened each light curve using the LIGHTKURVE tool, part of the publicly available Kepler/K2 community tools (Lightkurve Collaboration 2018). These flattened light curves were then searched systematically using the method set out in Osborn et al. (2016), searching for single-transit events up to $24 \mathrm{~h}$ in duration. We detected of the order of 1000 high signal-tonoise-ratio events per sector. We vet these candidate events further by checking for known systematics, known planets or eclipsing binaries, checking Gaia DR2 (Gaia Collaboration 2018) for blends and analysing the TESS full-frame images (FFIs) for asteroids and other external influences.

Using this process, TIC-238855958 was identified as a strong single-transit candidate in our search of the TESS Sector 1 data. TIC238855958 is a $T=10.23 \mathrm{mag}$ star located at $\mathrm{RA}=342.750156^{\circ}$ and Dec. $=-67.51508^{\circ}$. From the TESS Input Catalogue 8 (Stassun et al. 2018), TIC-238855958 is a $T_{\text {eff }}=6200 \mathrm{~K} \mathrm{~F} \mathrm{dwarf} \mathrm{with} \mathrm{a} \mathrm{radius}$ of $2.26 \mathrm{R}_{\odot}$. TIC-238855958 does not appear in subsequent TESS Sectors, so there was no possibility of further transits in the TESS data. We show the flattened difference imaging full-frame light curve for TIC-238855958 in Fig. 1. The single-transit event has a depth of $7750 \mathrm{ppm}$ and duration of $7 \mathrm{~h}$. Excluding the transit feature, the light curve of TIC-238855958 shows an RMS of 460 ppm, so the transit feature is clearly significant. No other stars around TIC238855958 show a similar transit feature at this epoch, helping rule out a spacecraft systematic. We see no evidence for any asteroid or

${ }^{1}$ https://filtergraph.com/tess_ffi/sector-01 other irregularity (including centroid offsets) in the full-frame pixel data that could be responsible for the single-transit event.

\section{TRANSIT PRECOVERY WITH WASP}

For each single-transit candidate we identify in TESS data using the method set out in Section 2, we cross-match the star with archival data from the Wide-Angle Search for Planets (WASP; Pollacco et al. 2006). WASP operates two survey instruments: one at the South African Astronomical Observatory (SAAO), South Africa, and another at the Observatorio del Roque de los Muchachos, La Palma. TIC-238855958 was observed for 3 consecutive observing seasons from 2010 to 2012 (1SWASPJ225059.97-673054.2) from the south station (27223 observations in total).

In order to search the archival photometric data for evidence of transit events, we use a template-matching algorithm. We first construct a best-fitting TESS template. Although we used the difference-imaging light curve of Oelkers \& Stassun (2018) for the detection (see Section 2), we found that for TIC-238855958 the light curve produced using the ELEANOR pipeline (Feinstein et al. 2019) was of slightly higher photometric quality. We thus adopted the ELEANOR light curve for our template matching. The ELEANOR light curve of TIC-238855958 was modelled using a Bayesian sampler provided through the PYTHON package, EMCEE, (Foreman-Mackey et al. 2013) using the transit model described in Section 6.2. We fixed the orbital period to $30 \mathrm{~d}$ and fitted only the transit epoch, $T_{0}$, the scaled orbital separation, $R_{1} / a$, the ratio of radii, $k=R_{2} / R_{1}$, the impact parameter, $b$, and the photometric zero-point, $z p$. Limb-darkening parameters were fixed and interpolated using effective surface temperature $\left(T_{\text {eff }}\right)$ from TESS Input Catalogue 8 assuming solar surface metalicity $([\mathrm{Fe} / \mathrm{H}])$ and surface gravity $(\log g)$. We ran 50 Markov chains for 10000 draws and found best-fitting parameters of $R_{1} / a=0.083, k=$ 0.080 , and $b=0.12$. This transit template was then used as a matched filter, fitting it to the WASP photometry at each time point in the data set and recording the $\chi^{2}$ statistic to quantify the goodness of fit as a function of transit mid-time. We first calculate the weighted mean $\left(w_{m}\right)$ of the full WASP data set and calculate $\chi_{\text {ref }}^{2}=\sum_{i}\left(m_{i}-w_{m}\right)^{2} / \sigma_{i}^{2}$, where $\sigma_{i}$ is the magnitude error attributed to each data point. The TESS template was centred at each point in the WASP light curve and calculated $\Delta \chi^{2}=\chi^{2}-\chi_{\text {ref }}^{2}$, where $\chi^{2}=\sum_{i}\left(m_{i}-t_{i}\right)^{2} / \sigma_{i}^{2}$ and $t_{i}$ is the centred TESS template at each point in the WASP light curve. Times where the template is well-matched to the data correspond to peaks in $\Delta \log \mathcal{L}=$ $-\Delta \chi^{2} / 2$. Validating these peaks required sufficient thresholding to ensure relatively small peaks in $\Delta \log \mathcal{L}$ caused by white and red photometric noise were not mistaken for transit-like events. We use an empirically determined threshold of $\Delta \log \mathcal{L}>35$ as our minimum threshold for selecting single-transit-event candidates.

For TIC-238855958, four significant peaks were identified in $\Delta \log \mathcal{L}>35$ corresponding to transit-like events in WASP photometry (events 1-4 ordered chronologically; see Fig. 2). Events 1-3 are likely real due to the number of in-transit data points and how well-matched the photometry is to the template. Event 4, which is only just above our threshold, contains only 7 in-transit data points, is likely spurious, and so was excluded. Indeed, the orbital ephemerides from global modelling (Section 6.2) confirm that only three nights of WASP observations have in-transit data (events 1-3). The maximum orbital period is the smallest elapsed time between transit events, which is $114.585 \mathrm{~d}$ between events 1 and 2 . Using the WASP and TESS photometry, we are able to determine that the 


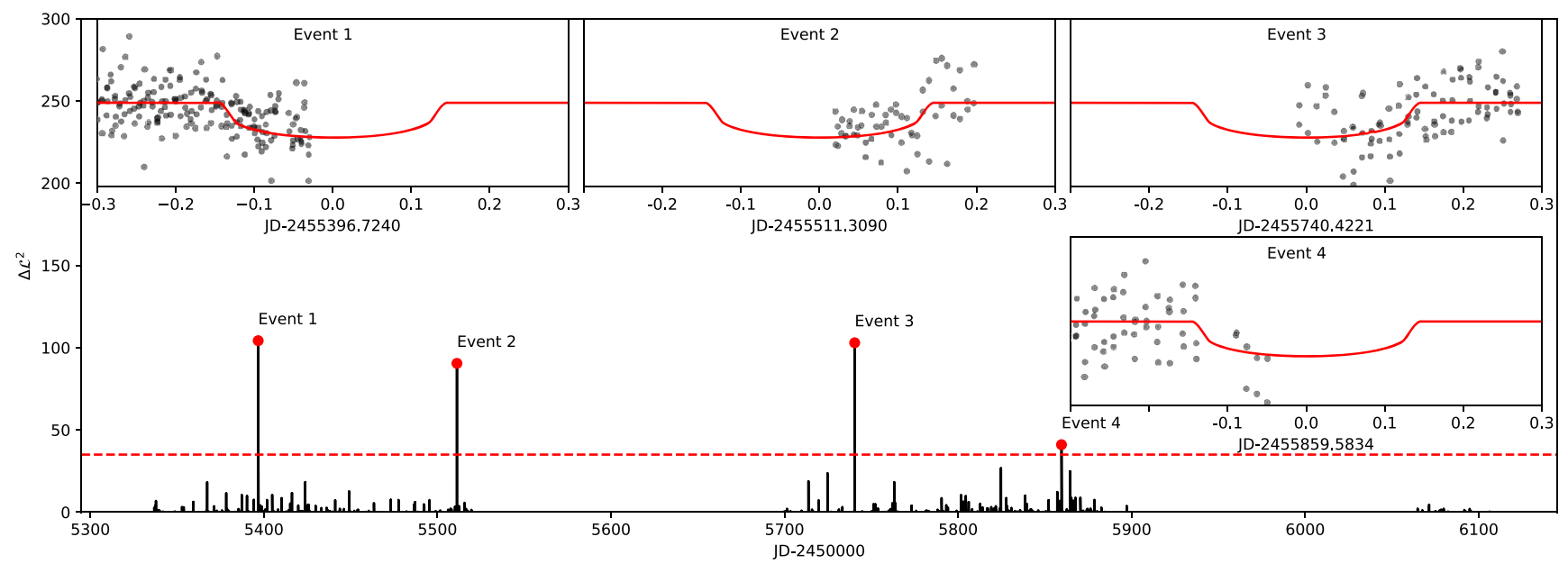

Figure 2. Results of template matching in archival WASP data for TIC-238855958. $\Delta \chi^{2}$ is plotted as a function of time with peaks labelled with red circles when they meet the threshold level (red dashed). The WASP light curve for each matching event is plotted as an inset panel, with the TESS template overlaid in red.

orbital period of TIC-238855958 must be one of three possible orbital periods: $P_{\text {orb }}[\mathrm{d}]=\{114.585,57.293,38.195\}$.

\section{NGTS TRANSIT DETECTION}

In order to test the set of three possible periods found using the WASP data in Section 3, we used the Next Generation Transit Survey (NGTS; Wheatley et al. 2018) telescopes located at the ESO Paranal Observatory in Chile. NGTS was designed for very high precision time-series photometry of stars, and thus is the perfect instrument to use for photometric follow-up of TESS single-transit candidates. The NGTS telescopes are robotic, so it is straightforward to schedule observations based on the possible periods of a given single-transit candidate. Each NGTS telescope has a field of view of 8 square degrees, providing sufficient reference stars for even the brightest TESS candidates. The telescopes have apertures of $20 \mathrm{~cm}$ and observe at a bandpass of 520-890 nm. Full details of the NGTS telescopes and cameras can be found in Wheatley et al. (2018).

We scheduled a single NGTS telescope to observe TIC238855958 on the night of 2019 Aug 23 in order to cover the next visible transit event assuming a $38 \mathrm{~d}$ orbital period. We observed TIC-238855958 for $9 \mathrm{~h}$ under photometric conditions with air mass $<2$. In total we obtain 2512 observations, each with exposure times of $10 \mathrm{~s}$. Data were reduced on-site using standard aperture photometry routines. The final light curve is presented in Fig. 3, and shows a robust detection of the transit event for TIC-238855958 that is consistent with the single event seen in the TESS data in Section 2 and the partial events from the WASP data set out in Section 3. This confirmed that the only possible orbital period for TIC-238855958 was the $38.195 \mathrm{~d}$ solution.

\section{SPECTROSCOPIC OBSERVATIONS}

In addition to the photometric follow-up of our TESS single transit-event candidates set out in Section 4, we also have an ongoing campaign to monitor these candidates spectroscopically using CORALIE - a fiber-fed échelle spectrograph installed on the 1.2-m Leonard Euler telescope at the ESO La Silla Observatory
(Queloz et al. 2001; Wilson et al. 2008). Candidates are vetted with a single observation to check for interlopers such as double lined eclipsing binary systems. Further observations are then taken spaced appropriately in time in order to determine the mass of the secondary companion.

Following the successful recovery of the orbital period of TIC238855958 using NGTS, we took eight spectroscopic observations of TIC-238855958 with CORALIE using an exposure time of $t_{\text {exp }}=600 \mathrm{~s}$. The spectra were reduced with the CORALIE standard reduction pipeline, and radial velocity measurements were obtained using standard cross-correlation techniques using numerical masks. We found a high amplitude radial velocity signal of $K$ $=7.01 \pm 0.05 \mathrm{~km} \mathrm{~s}^{-1}$ which was in phase with the photometric observation - see Fig. 4. This amplitude indicated that the companion to TIC-238855958 was in fact stellar in nature, and was also on a moderately eccentric orbit. We used these radial velocity measurements in our global modelling set out in Section 6.2. We compared the radial velocities to the bisector spans and found no evidence of correlation.

\section{ANALYSIS}

\subsection{Stellar atmospheric parameters}

We used wavelet analysis to extract atmospheric parameters from the co-added eight CORALIE spectroscopic observations of TIC238855958 (Section 5) using the methodology set out in Gill, Maxted \& Smalley (2018) and Gill et al. (2019). The co-added spectrum was re-sampled between 450 and $650 \mathrm{~nm}$ with $2^{17}$ values. The wavelet coefficients $W_{i=4-14, k}$ were calculated (see fig. 2 of Gill et al. 2018) and fitted against the same coefficients from model spectra in a Bayesian framework. We initiated 100 walkers and generated 100000 draws as a burn-in phase. We generated a further 100000 draws to sample the cumulative posterior probability distribution (PPD) for $T_{\text {eff }},[\mathrm{Fe} / \mathrm{H}], V \sin i$, and $\log g$. The wavelet method for CORALIE spectra can determine $T_{\text {eff }}$ to a precision of $85 \mathrm{~K},[\mathrm{Fe} / \mathrm{H}]$ to a precision of 0.06 dex and $V \sin i$ to a precision of $1.35 \mathrm{~km} \mathrm{~s}^{-1}$. TIC-238855958 has a projected rotation below $0.5 \mathrm{~km} \mathrm{~s}^{-1}$ and so we do not attribute any uncertainty to our measurement of $V \sin i$. 


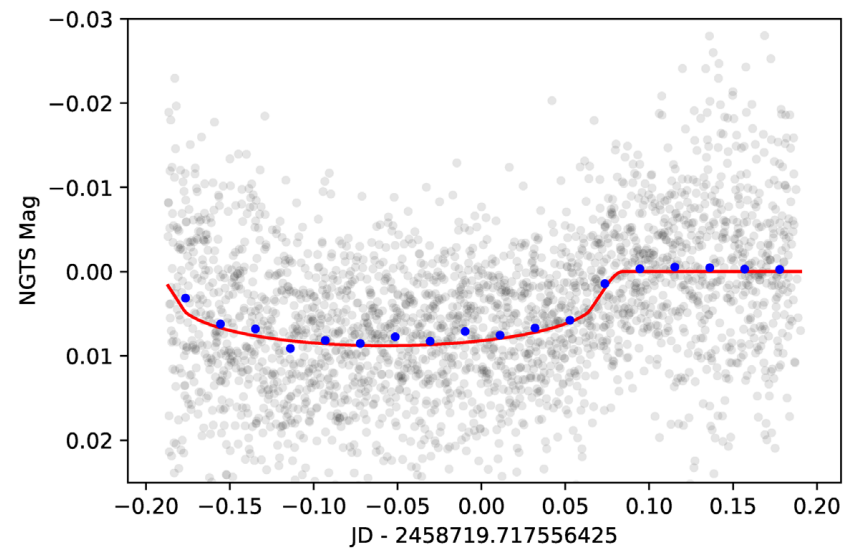

Figure 3. NGTS photometry of TIC-238855958 on the night of 2019 August 23. We plot the $30 \mathrm{~min}$ bins (blue) along with the best-fitting global model (red).
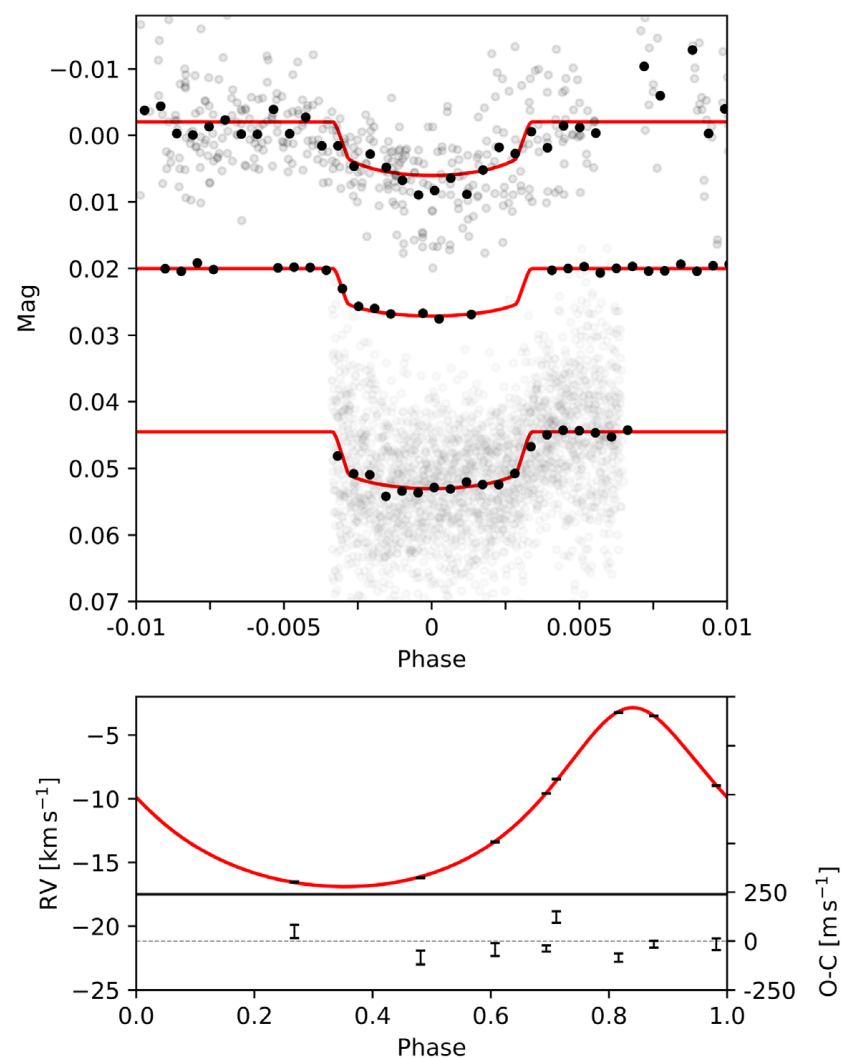

Figure 4. Orbital solution for TIC-238855958. Transit photometry from WASP (top), TESS (upper middle), and NGTS (lower middle) with corresponding best-fitting models (red). The darker points show all three light curves at $30 \mathrm{~min}$ cadence. The bottom panel shows CORALIE radial velocity measurements (black) with best-fitting model (red).

Measurements of $\log g$ from wavelet analysis are not reliable beyond confirming dwarf-like gravity ( $\log g \approx 4.5 \mathrm{dex})$. Subsequently, we fit the wings of the magnesium triplets with spectral synthesis by fixing $T_{\text {eff }},[\mathrm{Fe} / \mathrm{H}]$, and $V \sin i$ and changing $\log g$ until an acceptable fit was found. All our derived parameters for TIC-238855958 are set out in full in Table 1.
Table 1. Stellar atmospheric parameters, orbital solution, and physical properties of the TIC-238855958 system. Symmetric errors are reported with \pm and asymmetric errors are reported in brackets and correspond to the difference between the median and the 16th (lower value) and 84th (upper value) percentile.

\begin{tabular}{|c|c|}
\hline Parameter & Value \\
\hline \multicolumn{2}{|l|}{ Gaia } \\
\hline Source ID & 6391016653342250240 \\
\hline $\mathrm{BP}$ & $10.865563 \pm 0.01$ \\
\hline $\mathrm{RP}$ & $10.209588 \pm 0.01$ \\
\hline Parallax (mas) & $2.5025 \pm 0.0236$ \\
\hline \multicolumn{2}{|l|}{ Spectroscopy } \\
\hline $\mathrm{T}_{\mathrm{eff}}(\mathrm{K})$ & $6280 \pm 85$ \\
\hline $\log g(\operatorname{dex})$ & $4.01 \pm 0.13$ \\
\hline$\xi_{\mathrm{t}}\left(\mathrm{km} \mathrm{s}^{-1}\right)$ & $1.17 \pm 1.50$ \\
\hline$v_{\mathrm{mac}}\left(\mathrm{km} \mathrm{s}^{-1}\right)$ & $4.67 \pm 1.50$ \\
\hline$V \sin i\left(\mathrm{~km} \mathrm{~s}^{-1}\right)$ & $\leq 0.5$ \\
\hline$[\mathrm{Fe} / \mathrm{H}]$ & $0.20 \pm 0.06$ \\
\hline \multicolumn{2}{|l|}{ Orbital solution } \\
\hline $\mathrm{T}_{0}(\mathrm{JD})$ & $2458337.730695_{(1264)}^{(1701)}$ \\
\hline Period (d) & $38.195178_{(121)}^{(58)}$ \\
\hline$R_{1} / a$ & $0.0164_{(5)}^{(2)}$ \\
\hline$R_{2} / R_{1}$ & $0.0789_{(5)}^{(10)}$ \\
\hline$b$ & $0.034_{(10)}^{(89)}$ \\
\hline $\mathrm{h}_{1, \mathrm{WASP}}$ & $0.7387_{(7)}^{(12)}$ \\
\hline $\mathrm{h}_{2, \text { WASP }}$ & $0.2000_{(6)}^{(8)}$ \\
\hline $\mathrm{h}_{1, \mathrm{TESS}}$ & $0.8267_{(18)}^{(2)}$ \\
\hline $\mathrm{h}_{2, \mathrm{TESS}}$ & $0.2023_{(80)}^{(100)}$ \\
\hline $\mathrm{h}_{1, \mathrm{NGTS}}$ & $0.8214_{(18)}^{(1)}$ \\
\hline $\mathrm{h}_{2, \mathrm{NGTS}}$ & $0.2042_{(8)}^{(7)}$ \\
\hline$\sigma_{\mathrm{WASP}}$ & $0.00756_{(34)}^{(2)}$ \\
\hline$\sigma_{\mathrm{TESS}}$ & $0.00045_{(6)}^{(16)}$ \\
\hline$\sigma_{\mathrm{NGTS}}$ & $0.00804_{(22)}^{(1)}$ \\
\hline$K_{1}\left(\mathrm{~km} \mathrm{~s}^{-1}\right)$ & $7.013_{(36)}^{(49)}$ \\
\hline$f_{s}$ & $-0.038_{(22)}^{(9)}$ \\
\hline$f_{c}$ & $0.545_{(3)}^{(1)}$ \\
\hline$e$ & $0.298_{(4)}^{(1)}$ \\
\hline$\omega\left(^{\circ}\right)$ & $-3.9_{(2.1)}^{(0.9)}$ \\
\hline$V_{0}\left(\mathrm{~km} \mathrm{~s}^{-1}\right)$ & $-12.14_{(60)}^{(80)}$ \\
\hline $\mathrm{d} V_{0} / \mathrm{d} t\left(\mathrm{~km} \mathrm{~s}^{-1} \mathrm{~d}^{-1}\right)$ & $-0.003_{(2)}^{(1)}$ \\
\hline$J\left(\mathrm{~km} \mathrm{~s}^{-1}\right)$ & $0.054_{(2)}^{(102)}$ \\
\hline \multicolumn{2}{|l|}{ Physical properties } \\
\hline$M_{1}\left(\mathrm{M}_{\odot}\right)$ & $1.514 \pm 0.037$ \\
\hline$R_{1}\left(\mathrm{R}_{\odot}\right)$ & $2.159 \pm 0.037$ \\
\hline$M_{2}\left(\mathrm{M}_{\odot}\right)$ & $0.148 \pm 0.003$ \\
\hline$R_{2}\left(\mathrm{R}_{\odot}\right)$ & $0.171 \pm 0.003$ \\
\hline Age (Gyr) & $2.2 \pm 0.2$ \\
\hline
\end{tabular}

\subsection{Global modelling}

We collectively modelled WASP, TESS, and NGTS photometry with the CORALIE radial velocity measurements. Preliminary modelling of each photometric data set found consistent transit depths (to within $1 \sigma$ ) so we decided to fit a common transit depth ( $k=R_{2} / R_{1}$ ). Our model used the method described by Maxted (2016) to solve Kepler's equations and the analytical approximation 


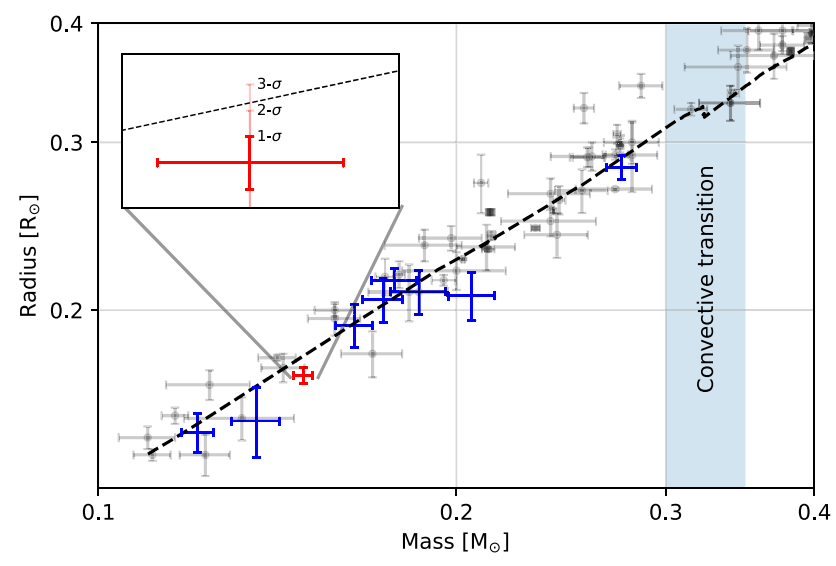

Figure 5. Mass-radius diagram for late M-dwarfs in eclipsing systems. We plot the M-dwarf companion of TIC-238855958 in red, M-dwarfs measured within the EBLM project in blue, and M-dwarfs with masses and radii known to better than 10 per cent (from table 4 of Chaturvedi et al. 2018, and references therein) in black. We overplot the best-fitting isochrone for TIC-238855958 (black-dashed).

presented by Maxted \& Gill (2019) to describe an object eclipsing a star with limb-darkening described by the power-2 law. We fitted the decorrelated limb-darkening parameters $h_{1}$ and $h_{2}$ from equations (1) and (2) of Maxted (2018). Following the suggestion by Maxted (2018), Gaussian priors were centred on interpolated values of $h_{1}$ and $h_{2}$ (from table 2 of Maxted 2018 via the PYCHEOPS python package) with widths of 0.003 and 0.046 , respectively. The similarity between NGTS and TESS transmission filters is such that they could be fitted with common limb-darkening priors. We used a different limb-darkening prior for the WASP filter which is bluer than TESS and NGTS.

Our model vector included $T_{0}$, the orbital period, $P, R_{1} / a$, $k=R_{2} / R_{1}, b$, independent values of the photometric zero-point, $z p, h_{1}$ and $h_{2}$ for each bandpass, the semi-amplitude, $K_{1}$, the radial velocity zero- of the primary star, $\gamma$, and the change radial velocity of the primary star with time, $\mathrm{d}(\gamma) / \mathrm{d} t$. Instead of fitting the argument of the periastron $(\omega)$ and the eccentricity $(e)$, we used $f_{c}=\sqrt{e} \cos \omega$ and $f_{s}=\sqrt{e} \sin \omega$ since these have a uniform prior probability distribution and are not strongly correlated with each other. We also include a jitter term added in quadrature to radial velocity uncertainties $(J)$ to account for spot activity, pulsations, and granulation which can introduce noise in to the radial velocity measurements (Ford 2006). This was added in quadrature to the uncertainties associated with each RV measurement. We fit a similar term for each photometric data set, $\sigma$, which was also added in quadrature. We sample parameter space using the Bayesian sampler described in Section 3. We ran 50 Markov chains for 100000 draws and discarded the first 50000 as a burn-in phase - visual checks ensured convergence was achieved well before the $50000^{\text {th }}$ draw. We selected the trial step with the highest value of log-likelihood as the measurement for each parameter. Asymmetric uncertainties were calculated using the differences between the measurement and the 16th and 84th percentiles of the PPD. Fitted parameters are reported in Table 1 and shown in Fig. 4.

\subsection{Physical properties}

We used the ISOCHRONES python package (Morton 2015) to measure the physical properties of the host star. Our vector of model parameters included the Gaia magnitudes $B P$ and $G P$ and parallax with Gaussian priors centred on values reported from GAIA DR2 (Gaia Collaboration 2018) with widths of 0.01 mag, $\mathrm{T}_{\text {eff }}, \log g$, and $[\mathrm{Fe} / \mathrm{H}]$ with prior centres and width equivalent to values and errors reported in Table 1, respectively. We use EMCEE to sample the posterior distributions of each parameters. We discarded 10000 draws as a burn-in phase before drawing the sample number of post burn-in draws used in Section 6.2. The PPD for the physical parameters associated with the fit $\left(M_{1}\right.$, $R_{1}$, age) were used to calculate the physical properties of the Mdwarf. We calculated the PPD for $R_{2}$ by multiplying the PPDs for $k$ and $R_{1}$. Calculating $M_{2}$ required solving the spectroscopic mass function,

$f(M)=\frac{\left(M_{2} \sin i\right)^{3}}{\left(M_{\star}+M_{2}\right)^{2}}=\left(1-e^{2}\right)^{3 / 2} \frac{P K_{1}^{3}}{2 \pi G}$,

where $G$ is the gravitational constant. For each step in the respective samplers, we evaluated the left-hand side of equation (1) and solved for $M_{2}$ using the corresponding value of $M_{\star}$. We assumed both stars are coeval.

\section{DISCUSSION AND CONCLUSIONS}

Our global modelling shows TIC-238855958 to be an eccentric, long period (38.2 d), F-M eclipsing binary. The primary F-star appears to have turned-off the main sequence, but has made little progress through the red-giant branch phase. Due to its mass $\left(M_{1}=\right.$ $\left.1.514 \pm 0.037 \mathrm{M}_{\odot}\right)$, the primary star is set to transition through the post-main-sequence blue hook that marks the passage from central hydrogen burning to shell burning. The M-dwarf has a mass of $M_{2}=0.148 \pm 0.003 \mathrm{M}_{\odot}$ and a radius of $R_{2}=0.171 \pm 0.003 \mathrm{R}_{\odot}$, making it one of the best characterized low-mass stars in terms of its mass and radius. This is shown in Fig. 5 where we compare the mass and radius of the M-dwarf with other well measured low-mass stars. We find it to be $2.3 \sigma$ smaller than expected compared with evolutionary models, see Fig. 5. This is consistent with J2308-46 and $\mathrm{J} 1847+39$, which are deflated by at least $1 \sigma$ (Gill et al. 2019). In general, M-dwarfs appear to be systematically inflated and cooler than predicted from models of stellar evolution (see fig. 9 of Lubin et al. 2017). It would be interesting to measure the secondary eclipse depth for this system and determine the temperature of the transiting M-dwarf and compare its luminosity with evolutionary models. Using PHOENIX model spectra (Husser et al. 2013) the transiting M-dwarf is fainter by a factor of $\sim 1000$ relative to the evolved primary star across the TESS transmission bandpass. We estimate a surface brightness ratio $S=0.00019$ corresponding to a secondary transit depth $<2 \mathrm{ppm}$. This is far below the out of transit RMS of $460 \mathrm{ppm}$ from the discovery TESS light curve and we do not expect to see a secondary eclipse should TIC-238855958 be observed in the TESS extended mission. If a secondary eclipse was observable, it would occur at phase 0.687 .

TIC-238855958 is the first success of the NGTS Mono-transit Working Group and its discovery paves the way to characterizing long-period eclipsing systems. This work highlights the pivotal role archival photometric data bases play in the recovery of orbital periods in conjunction with current photometric and spectroscopic instruments. Although this system is a long-period eclipsing binary, the transit depth is consistent with a giant planet around a solar-type star and so our method will be just as efficient at finding long-period transiting exoplanets. 


\section{ACKNOWLEDGEMENTS}

The NGTS facility is operated by the consortium institutes with support from the UK Science and Technology Facilities Council (STFC) under projects ST/M001962/1 and ST/S002642/1. Contributions at the University of Geneva by FB, LN, ML, OT, and SU were carried out within the framework of the National Centre for Competence in Research 'PlanetS' supported by the Swiss National Science Foundation (SNSF). The contributions at the University of Warwick by PJW, RGW, DLP, DJA, DRA, SG, and TL have been supported by STFC through consolidated grants ST/L000733/1 and ST/P000495/1. DJA acknowledges support from the STFC via an Ernest Rutherford Fellowship (ST/R00384X/1). The contributions at the University of Leicester by MGW and MRB have been supported by STFC through consolidated grant ST/N000757/1. SLC acknowledges support from the STFC via an Ernest Rutherford Fellowship (ST/R003726/1). JSJ is supported by funding from Fondecyt through grant 1161218 and partial support from CATA-Basal (PB06, Conicyt). ACC acknowledges support from the Science and Technology Facilities Council (STFC) consolidated grant number ST/R000824/1. MNG acknowledges support from the Juan Carlos Torres Fellowship. ACh acknowledges the support of the DFG priority program SPP 1992 'Exploring the Diversity of Extrasolar Planets' (RA 714/13-1).

\section{REFERENCES}

Chaturvedi P., Sharma R., Chakraborty A., Anandarao B. G., Prasad N. J. S. S. V., 2018, AJ, 156, 27

Cooke B. F., Pollacco D., West R., McCormac J., Wheatley P. J., 2018, A\&A, 619, A175

Feinstein A. D. et al., 2019, PASP, 131, 094502

Ford E. B., 2006, ApJ, 642, 505

Foreman-Mackey D., Hogg D. W., Lang D., Goodman J., 2013, PASP, 125, 306

Gaia Collaboration, 2018, A\&A, 616, A1

Gill S., Maxted P. F. L., Smalley B., 2018, A\&A, 612, A111

Gill S. et al., 2019, A\&A, 626, A119

Günther M. N. et al., 2019, Nat. Astron., 420

Huang C. X. et al., 2018, ApJ, 868, L39

Husser T.-O., Wende-von Berg S., Dreizler S., Homeier D., Reiners A., Barman T., Hauschildt P. H., 2013, A\&A, 553, A6

Lightkurve Collaboration, 2018, Astrophysics Source Code Library, record ascl: 1812.013
Lubin J. B. et al., 2017, ApJ, 844, 134

Maxted P. F. L., 2016, A\&A, 591, A111

Maxted P. F. L., 2018, A\&A, 616, A39

Maxted P. F. L., Gill S., 2019, A\&A, 622, A33

Morton T. D., 2015, Astrophysics Source Code Library, record ascl: 1503.010

Nielsen L. D. et al., 2019, A\&A, 623, A100

Oelkers R. J., Stassun K. G., 2018, AJ, 156, 132

Osborn H. P. et al., 2016, MNRAS, 457, 2273

Pollacco D. L. et al., 2006, PASP, 118, 1407

Queloz D. et al., 2001, A\&A, 379, 279

Ricker G. R. et al., 2015, J. Astron. Telesc. Instrum. Syst., 1, 014003

Stassun K. G. et al., 2018, AJ, 156, 102

Vanderburg A. et al., 2019, ApJ, 881, L19

Villanueva Steven J., Dragomir D., Gaudi B. S., 2019, AJ, 157, 84

Wheatley P. J. et al., 2018, MNRAS, 475, 4476

Wilson D. M. et al., 2008, ApJ, 675, L113

${ }^{1}$ Department of Physics, University of Warwick, Gibbet Hill Road, Coventry CV4 7AL, UK

${ }^{2}$ Centre for Exoplanets and Habitability, University of Warwick, Gibbet Hill Road, Coventry CV4 7AL, UK

${ }^{3}$ Observatoire de Genève, Université de Genève, $51 \mathrm{Ch}$. des Maillettes, CH-1290 Sauverny, Switzerland

${ }^{4}$ Space Research Institute, Austrian Academy of Sciences, Schmiedlstr. 6, A-8042 Graz, Austria

${ }^{5}$ School of Physics and Astronomy, University of Leicester, Leicester LE1 $7 R H, U K$

${ }^{6}$ Centre for Exoplanet Science, SUPA, School of Physics and Astronomy, University of St Andrews, St Andrews KY16 9SS, UK

${ }^{7}$ Center for Astronomy and Astrophysics, TU Berlin, Hardenbergstr. 36, D-10623 Berlin, Germany

${ }^{8}$ Department of Physics, and Kavli Institute for Astrophysics and Space Research, Massachusetts Institute of Technology, Cambridge, MA 02139, USA

${ }^{9}$ Astrophysics Group, Keele University, Staffordshire ST5 5BG, UK

${ }^{10}$ Departamento de Astronomía, Universidad de Chile, Camino El Observatorio 1515, Las Condes, Santiago, Chile

${ }^{11}$ Centro de Astrofísica y Tecnologías Afines (CATA), Casilla 36-D, Santiago, Chile

${ }^{12}$ Instituto de Astronomía, Universidad Católica del Norte, Angamos 0610, 1270709 Antofagasta, Chile

${ }^{13}$ Institute of Planetary Research, German Aerospace Center, Rutherfordstrasse 2, D-12489 Berlin, Germany

This paper has been typeset from a $\mathrm{T}_{\mathrm{E}} \mathrm{X} / \mathrm{LT} \mathrm{E} \mathrm{X}$ file prepared by the author. 